Pacific

Journal of

Mathematics

THE UNTWISTING NUMBER OF A KNOT

KENAN INCE

Volume 283 No. 1

July 2016 


\title{
THE UNTWISTING NUMBER OF A KNOT
}

\author{
KENAN INCE \\ Dedicated to Tim Cochran
}

\begin{abstract}
The unknotting number of a knot is the minimum number of crossings one must change to turn that knot into the unknot. The algebraic unknotting number is the minimum number of crossing changes needed to transform a knot into an Alexander polynomial-one knot. We work with a generalization of unknotting number due to Mathieu and Domergue, which we call the untwisting number. The untwisting number is the minimum number (over all diagrams of a knot) of right- or left-handed twists on even numbers of strands of a knot, with half of the strands oriented in each direction, necessary to transform that knot into the unknot. We show that the algebraic untwisting number is equal to the algebraic unknotting number. However, we also exhibit several families of knots for which the difference between the unknotting and untwisting numbers is arbitrarily large, even when we only allow twists on a fixed number of strands or fewer.
\end{abstract}

\section{Introduction}

It is a natural knot-theoretic question to seek to measure "how knotted up" a knot is. One such "knottiness" measure is given by the unknotting number $u(K)$, the minimum number of crossings, taken over all diagrams of $K$, one must change to turn $K$ into the unknot. By a crossing change we shall mean one of the two local moves on a knot diagram given in Figure 1.

This invariant is quite simple to define but has proven itself very difficult to master. Fifty years ago, Milnor conjectured that the unknotting number for the $(p, q)$-torus knot was $\frac{1}{2}(p-1)(q-1)$; only in 1993, in two celebrated papers, did Kronheimer and Mrowka [1993; 1995] prove this conjecture true. Hence, it is desirable to look at variants of unknotting number which may be more tractable. One natural variant (due to Murakami [1990]) is the algebraic unknotting number $u_{a}(K)$, the minimum number of crossing changes necessary to turn a given knot into an Alexander polynomial-one knot. Alexander polynomial-one knots are significant because they "look like the unknot" to classical invariants, knot invariants derived

MSC2010: 57M25, 57M27.

Keywords: knot, knot theory, unknotting, unknotting number, topology. 


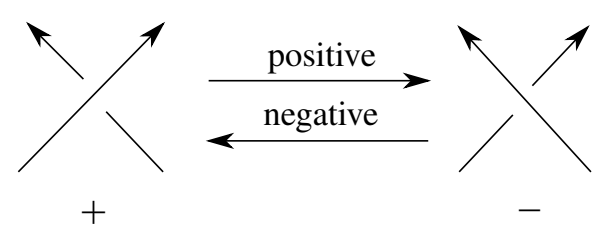

Figure 1. A positive and negative crossing change.

from the Seifert matrix. It is obvious that $u_{a}(K) \leq u(K)$ for any knot $K$, and there exist knots such that $u_{a}(K)<u(K)$ (for instance, any nontrivial knot with trivial Alexander polynomial).

Mathieu and Domergue [1988] defined another generalization of unknotting number. Livingston [2002] worked with this definition. He described it as follows:

"One can think of performing a crossing change as grabbing two parallel strands of a knot with opposite orientation and giving them one full twist. More generally, one can grab $2 k$ parallel strands of $K$ with $k$ of the strands oriented in each direction and give them one full twist."

Following Livingston, we call such a twist a generalized crossing change. We describe in Section 2A how a crossing change may be encoded as a \pm 1 -surgery on a nullhomologous unknot $U \subset S^{3}-K$ bounding a disk $D$ such that $D \cap K=2$ points. From this perspective, a generalized crossing change is a relaxing of the previous definition to allow $D \cap K=2 k$ points for any $k$, provided $\operatorname{lk}(K, U)=0$ (see Figure 2). In particular, any knot can be unknotted by a finite sequence of generalized crossing changes.

One may then naturally define the untwisting number $t u(K)$ to be the minimum length, taken over all diagrams of $K$, of a sequence of generalized crossing changes beginning at $K$ and resulting in the unknot. By $t u_{p}(K)$, we will denote the minimum number of twists on $2 p$ or fewer strands needed to unknot $K$; notice that $t u_{1}(K)=u(K)$ and that

$$
t u \leq \cdots \leq t u_{p+1} \leq t u_{p} \leq \cdots \leq t u_{1}=u .
$$
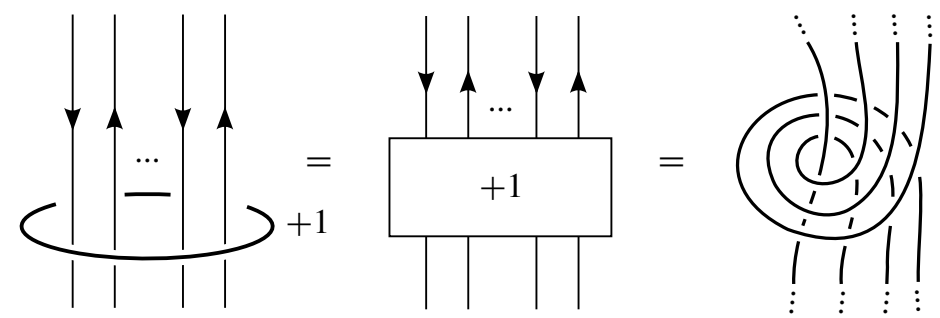

Figure 2. A right-handed, or positive, generalized crossing change. 


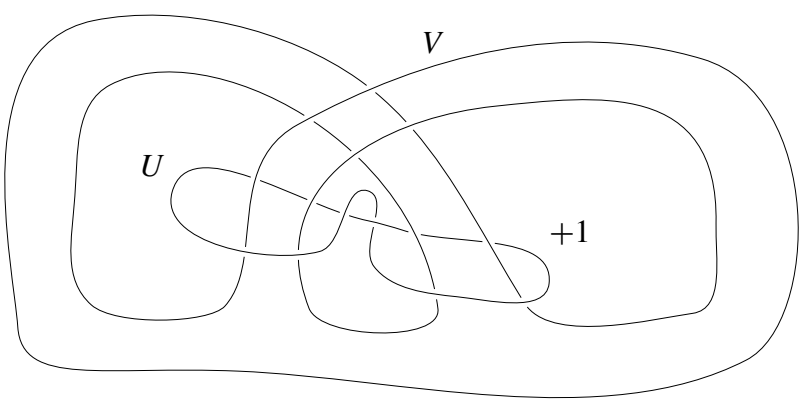

Figure 3. The generalized crossing change for $V \subset S^{3}$ which results in a knot $K \subset S^{3}$ with $t u(K) \neq u(K)$.

The algebraic untwisting number $t u_{a}(K)$ is the minimum number of generalized crossing changes, taken over all diagrams of $K$, needed to transform $K$ into an Alexander polynomial-one knot. It is clear that $t u_{a}(K) \leq t u(K)$ for all knots $K$.

It is natural to ask how $t u(K)$ and $u(K)$ are related. We show that these invariants are "algebraically the same" in the following sense:

Theorem 1.1. For any knot $K \subset S^{3}, t u_{a}(K)=u_{a}(K)$.

Therefore, $t u$ and $u$ cannot be distinguished by classical invariants. By using the Jones polynomial, which is not a classical invariant, we can show that $t u$ and $u$ are not equal in general:

Theorem 1.2. Let $K$ be the image of $V \subset S^{3}$ in the manifold $M \cong S^{3}$ resulting from +1 -surgery on the unknot $U \subset S^{3}$ shown in Figure 3. Then $t u(K)=1$ but $u(K)>1$.

Furthermore, using the fact that the absolute value of the Ozsváth-Szabó $\tau$ invariant is a lower bound on unknotting number, we show in Section $5 \mathrm{~A}$ that the difference $u-t u_{p}$ can be arbitrarily large, and thus so can the difference $u-t u$. Throughout this paper, $K_{p, q}$ will denote the $(p, q)$-cable of the knot $K$, where $p$ denotes the longitudinal winding and $q$ the meridional winding.

Theorem 1.3. Let $K$ be a knot in $S^{3}$ such that $u(K)=1$. If $\tau(K)>0$ and $p, q>0$, then

$$
u\left(K_{p, q}\right)-t u_{p}\left(K_{p, q}\right) \geq p-1 .
$$

In particular, if we take $q=1$, then $t u_{p}\left(K_{p, q}\right)=1$, while $u\left(K_{p, q}\right) \geq p$.

It may seem that the above examples are "cheating" in some sense, as in each of them the number of strands of $K$ passing through the \pm 1 -framed unknot $U$ in the generalized crossing change diagram is increasing along with $u(K)$. The following theorem shows that $u(K)$ can be arbitrarily larger than $t u(K)$ even when we restrict to doing $q$-generalized crossing changes for any fixed integer $q \geq 1$. 
Theorem 1.4. For any knot $K$ with $u(K)=1$ and $\tau(K)>0$, the infinite family of knots $J_{p}^{q}:=\#^{p} K_{q, 1}$ satisfies

$$
u\left(J_{p}^{q}\right)-t u_{q}\left(J_{p}^{q}\right) \geq p
$$

for any integers $p>1, q>0$.

So far, all of the families of knots we have worked with are quite complicated, in the sense that they are $(p, q)$-cables for large $p$ or connected sums of such cables. One may wonder whether it is possible to find a "simpler" knot $K$ for which $t u(K)<u(K)$. One measure of "knot simplicity" is topological sliceness; a knot $K$ is topologically slice if there exists a locally flat disk $D \subset B^{4}$ such that $\partial D=K \subset S^{3}=\partial B^{4}$.

Theorem 1.5. For any knot $K$ with $\tau(K)>0$, let $D_{+}(K, 0)$ denote the positiveclasped, untwisted Whitehead double of $K$. Then the knots $S_{p}^{q}:=\#^{p}\left(D_{+}(K, 0)\right)_{q, 1}$ are topologically slice and satisfy

$$
u\left(S_{p}^{q}\right)-t u_{q}\left(S_{p}^{q}\right) \geq p
$$

for all integers $p>0, q>0$.

This paper is organized as follows. First, we will review the operations of Dehn surgery on knots and knot cabling and define the untwisting number more precisely. Next, we will give some background on the Blanchfield form which is necessary to prove that $t u_{a}=u_{a}$. Finally, we will prove that each of the above families of knots gives arbitrarily large gaps between $u$ and $t u$.

Convention. In this paper, all manifolds are assumed to be compact, orientable, and connected.

\section{Preliminaries}

2A. Dehn surgery. In this section, we will describe the operation of Dehn surgery on knots.

Definition 2.1. Let $K \subset S^{3}$ be an oriented knot and $U \subset S^{3}$ be an unknot with $\operatorname{lk}(K, U)=0$. Let $W$ be a closed tubular neighborhood of $U$ in $S^{3}$. Let $\lambda$ be a longitude of $W$, and let $\mu$ be a meridian of $W$ such that $\operatorname{lk}(\mu, \lambda)=1$. The 3-manifold

$$
M=\left(S^{3}-\stackrel{\circ}{W}\right) \cup_{h} W,
$$

where $h: \partial W \rightarrow \partial W$ is a homeomorphism taking a meridian of $W$ onto $\pm \mu+\lambda \subset W$, is the result of \pm 1 -surgery on $U$, and $U$ is said to be \pm 1 -framed. In this situation, we define a generalized crossing change diagram for $K$ to be a diagram of the link $K \cup U$ with the number \pm 1 written next to $U$, indicating that $U$ is \pm 1 -framed. Figure 3 is an example of a generalized crossing change diagram for the unknot $V$. 


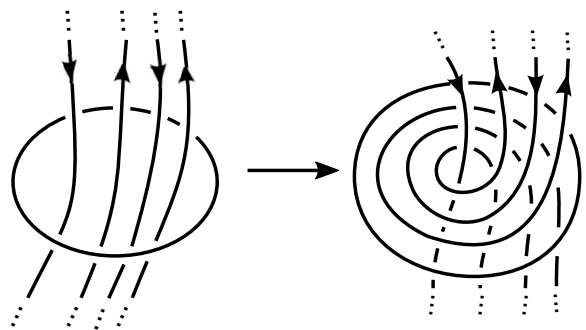

Figure 4. A right-handed twist about an unknotted component.

In the general case, note that the complement of $\stackrel{\circ}{N} \supset U$ in $S^{3}$ is a solid torus, which we may modify with a meridional twist. This alters $K$ as follows: if $D$ is a disk bounded by $U$ such that $k$ strands of $K$ pass through $D$ in straight segments, then each of the $k$ straight pieces is replaced by a helix which screws through a neighborhood of $D$ in the right-hand sense (see Figure 4).

If $U$ is -1 -framed, the knot obtained by erasing $U$ and twisting the strands of $K$ that pass through $U$ as in Figure 4 represents the image of $K$ under the -1 -surgery on $U$ [Rolfsen 1976]. If instead $U$ has framing +1 , the knot obtained by erasing $U$ and giving $K$ a left-handed meridional twist represents the image of $K$ under the +1 -surgery on $U$. The process of performing a 干-meridional twist on the complement of a \pm 1 -framed unknot $U$, then erasing $U$ from the resulting diagram, is called a blow-down on $U$. The inverse process of introducing an unknotted component $U$ to a surgery diagram consisting of a knot $K$, then performing a \pm -meridional twist on the complement of $U$ to link it with $K$, is known as a blow-up on $U$ and results in a diagram consisting of $K$ and the $\mp 1$-framed unknot $U$, where $\operatorname{lk}(K, U)=0$.

Now, it can be easily verified that blowing down the +1 -framed unknot on the left side of Figure 5 transforms the crossing labeled + into the crossing labeled - . The inverse process of introducing an unknot to the right side of Figure 5 and performing a --meridional twist on its complement yields the positive crossing.

2B. Untwisting number. We define a \pm -generalized crossing change on $K$ as the process of blowing down the \pm 1 -framed unknot in a generalized crossing change diagram for $K$. In this situation, $K$ must pass through $U$ an even number of times, for otherwise $\operatorname{lk}(K, U) \neq 0$. If at most $2 p$ strands of $K$ pass through $U$ in a

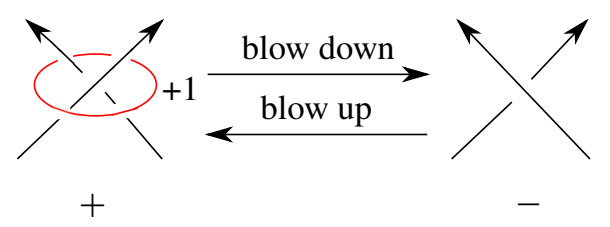

Figure 5. Crossing changes as blow-downs of \pm 1 -framed unknots. 
generalized crossing change diagram, we may call the associated \pm -generalized crossing change a $\pm p$-generalized crossing change on $K$.

The result of $a \pm$-generalized crossing change on $K$ is defined to be the image of $K$ under the blow-down. The untwisting number $t u(K)$ of $K$ is the minimum length of a sequence of generalized crossing changes on $K$ such that the result of the sequence is the unknot, where we allow ambient isotopy of the diagram in between generalized crossing changes. Note that by the reasoning on page 58 of [Adams 1994], this definition is equivalent to taking the minimum length, over all diagrams of $K$, of a sequence of generalized crossing changes beginning with a fixed diagram of $K$ such that the result of the sequence is the unknot, where we do not allow ambient isotopy of the diagram in between generalized crossing changes.

For $p=1,2,3, \ldots$, we define the $p$-untwisting number $t u_{p}(K)$ to be the minimum length of a sequence of $\pm p$-generalized crossing changes on $K$ resulting in the unknot, where we allow ambient isotopy of the diagram in between generalized crossing changes.

It follows immediately that we have the chain of inequalities

$$
t u(K) \leq \cdots \leq t u_{p+1}(K) \leq t u_{p}(K) \leq \cdots \leq t u_{2}(K) \leq t u_{1}(K)=u(K) .
$$

2C. Cabling. In this section, we define satellite and cable knots.

Definition 2.2. A closed subset $X$ of a solid torus $V \cong S^{1} \times D^{2}$ is called geometrically essential in $V$ if $X$ intersects every PL meridional disk in $V$.

Let $P \subset V \subset S^{3}$ be a knot which is geometrically essential in an unknotted solid torus $V$. Let $C \subset S^{3}$ be another knot and let $V_{1}$ be a tubular neighborhood of $C$ in $S^{3}$. Let $h: V \rightarrow V_{1}$ be a homeomorphism and let $K$ be $h(P)$. Then $P$ is called the pattern for the knot $K, C$ is the companion of $K$, and $K$ is called a satellite of $C$ with pattern $P$, or just a satellite knot for short.

If the homeomorphism $h$ takes the preferred longitude and meridian of $V$, respectively, to the preferred longitude and meridian of $V_{1}$, then $h$ is said to be faithful. If $P$ is the $(p, q)$-torus knot just under $\partial V$ and $h$ is faithful, then $K$ is called the $(p, q)$-cable based on $C$, denoted $C_{p, q}$, or simply a cable knot.

Throughout this paper, we will denote the $(p, q)$-torus knot by $U_{p, q}$ since it is the $(p, q)$-cable of the unknot $U$.

2D. The Blanchfield form. Let $K \subset S^{3}$ be a knot. By $\Lambda$ we shall denote the ring $\mathbb{Z}\left[t^{ \pm 1}\right]$, and by $\Omega$ we will denote the field $\mathbb{Q}(t)$.

Twisted homology, cohomology groups, and Poincaré duality. Following [Borodzik and Friedl 2014], let $X$ be a manifold with infinite cyclic first homology, and fix a choice of isomorphism of $H_{1}(X)$ with the infinite cyclic group generated by the indeterminate $t$. Let $\pi: \widetilde{X} \rightarrow X$ be the infinite cyclic cover of $X$. Given a 
submanifold $Y$ of $X$, let $\tilde{Y}=\pi^{-1}(Y)$. Since $\mathbb{Z}$ is the deck transformation group of $\widetilde{X}, \Lambda$ acts on the relative chain group $C_{*}(\widetilde{X}, \widetilde{Y} ; \mathbb{Z})$. If $N$ is any $\Lambda$-module, we may define

$$
H^{*}(X, Y ; N):=H_{*}\left(\operatorname{Hom}_{\Lambda}\left(C_{*}(\tilde{X}, \tilde{Y} ; \mathbb{Z}), N\right)\right)
$$

and

$$
\left.H_{*}(X, Y ; N):=H_{*}\left(\overline{C_{*}(\tilde{X}, \tilde{Y} ; \mathbb{Z}}\right) \otimes_{\Lambda} N\right) .
$$

Here, if $H$ is any $\Lambda$-module, $\bar{H}$ denotes the module with the involuted $\Lambda$-structure: multiplication by $p(t) \in \Lambda$ in $\bar{H}$ is the same as multiplication by $p\left(t^{-1}\right)$ in $H$. When $Y=\varnothing$, we just write $H_{*}(X ; N)$ or $H^{*}(X ; N)$.

Since $\Omega:=\mathbb{Q}(t)$ is flat over $\Lambda$, we have isomorphisms

$$
H_{*}(X, Y ; \Omega) \cong H_{*}(X, Y ; \Lambda) \otimes_{\Lambda} \Omega
$$

and

$$
H^{*}(X, Y ; \Omega) \cong H^{*}(X, Y ; \Lambda) \otimes_{\Lambda} \Omega \text {. }
$$

If $X$ is an $n$-manifold, and $N$ is a $\Lambda$-module, Poincaré duality gives $\Lambda$-module isomorphisms

$$
H_{i}(X, \partial X ; N) \cong H^{n-i}(X ; N) .
$$

The Blanchfield form. As above, let $\Lambda=\mathbb{Z}\left[t, t^{-1}\right]$ and $\Omega=\mathbb{Q}(t)$. Let $A$ be an $n \times n$ invertible hermitian matrix with entries in $\Lambda$. We define $\lambda(A)$ to be the pairing

$$
\lambda(A): \Lambda^{n} / A \Lambda^{n} \times \Lambda^{n} / A \Lambda^{n} \rightarrow \Omega / \Lambda
$$

sending the pair of column vectors $(a, b)$ to $\bar{a}^{t} A^{-1} b$. Note that $\lambda(A)$ is a nonsingular, hermitian pairing.

Let $X(K)=S^{3}-N(K)$ denote the exterior of $K$. Consider the sequence of maps

$$
\begin{aligned}
\Phi: H_{1}(X(K) ; \Lambda) & \stackrel{\pi_{*}}{\longrightarrow} H_{1}(X(K), \partial X(K) ; \Lambda) \\
& \stackrel{\mathrm{PD}}{\longrightarrow} H^{2}(X(K) ; \Lambda) \stackrel{\delta}{\longleftarrow} H^{1}(X(K) ; \Omega / \Lambda) \\
& \stackrel{\mathrm{ev}}{\longrightarrow} \overline{H o m}_{\lambda}\left(H_{1}(X(K) ; \Lambda), \Omega / \Lambda\right) .
\end{aligned}
$$

Here $\pi_{*}$ is induced by the quotient map $C(X) \rightarrow C(X) / C(\partial X)$, PD is the Poincaré duality map, $\delta$ is from the long exact sequence in cohomology obtained from the coefficients $0 \rightarrow \Lambda \rightarrow \Omega \rightarrow \Omega / \Lambda \rightarrow 0$, and ev is the Kronecker evaluation map. It is well known (see [Hillman 2012, Section 2] for details) that $\pi_{*}$ and $\delta$ are isomorphisms, PD is the Poincaré duality isomorphism, and ev is also an isomorphism by the universal coefficient spectral sequence (see [Levine 1977, Theorem 2.3] for details on the universal coefficient spectral sequence). Thus, the 
above maps define a nonsingular pairing

$$
\begin{aligned}
\lambda(K): H_{1}(X(K) ; \Lambda) \times H_{1}(X(K) ; \Lambda) & \rightarrow \Omega / \Lambda, \\
(a, b) & \mapsto \Phi(a)(b),
\end{aligned}
$$

called the Blanchfield pairing of $K$. This pairing is hermitian.

Now, let $V$ be any $2 k \times 2 k$ matrix which is $S$-equivalent to a Seifert matrix for $K$. Recall that $V-V^{T}$ is antisymmetric with determinant \pm 1 . It is well known that, perhaps after replacing $V$ by $P V P^{T}$ for some $P \in \mathrm{GL}_{2 k}(\mathbb{Z})$,

$$
V-V^{T}=\left(\begin{array}{cc}
0 & -I_{k} \\
I_{k} & 0
\end{array}\right)
$$

where $I_{k}$ denotes the $k \times k$ identity matrix. We define $A_{K}(t)$ to be the matrix

$$
\left(\begin{array}{cc}
\left(1-t^{-1}\right)^{-1} I_{k} & 0 \\
0 & I_{k}
\end{array}\right) V\left(\begin{array}{cc}
I_{k} & 0 \\
0 & (1-t) I_{k}
\end{array}\right)+\left(\begin{array}{cc}
I_{k} & 0 \\
0 & \left(1-t^{-1}\right) I_{k}
\end{array}\right) V^{T}\left(\begin{array}{cc}
(1-t)^{-1} I_{k} & 0 \\
0 & I_{k}
\end{array}\right) .
$$

Using (2-2), we can write

$$
V=\left(\begin{array}{cc}
B & C+I \\
C^{T} & D
\end{array}\right)
$$

One may then compute, as in the proof of [Borodzik and Friedl 2015, Lemma 2.2], that

$$
A_{K}(1)=\left(\begin{array}{rr}
B & -I_{k} \\
-I_{k} & 0
\end{array}\right)
$$

Thus, the matrix $A_{K}(t)$ is a hermitian matrix defined over $\Lambda$, and $\operatorname{det}\left(A_{K}(1)\right)=(-1)^{k}$.

Proposition 2.3 [Borodzik and Friedl 2015, Proposition 2.1]. Let $K$ be a knot and $A_{K}(t)$ be as above. Then $\lambda\left(A_{K}(t)\right)$ is isometric as a sesquilinear form to $\lambda(K)$.

2E. The twisted intersection pairing. Let $W$ be a topological 4-manifold with boundary $M$ such that $\pi_{1}(W)=\mathbb{Z}$. Consider the maps

$$
H_{2}(W ; \Lambda) \stackrel{\pi_{*}}{\longrightarrow} H_{2}(W, M ; \Lambda) \stackrel{\mathrm{PD}}{\longrightarrow} H^{2}(W ; \Lambda) \stackrel{\mathrm{ev}}{\longrightarrow} \overline{\operatorname{Hom}_{\Lambda}\left(H_{2}(W ; \Lambda), \Lambda\right)},
$$

where the first map is induced by the quotient, the second map is Poincare duality, and the third map is the Kronecker evaluation map. The second and third maps are obviously isomorphisms, and the first map is an isomorphism by the long exact sequence of the pair $(W, M)$. Hence this composition defines a pairing

$$
H_{2}(W ; \Lambda) \times H_{2}(W ; \Lambda) \rightarrow \Lambda,
$$

which we call the twisted intersection pairing on $W$. 


\section{Algebraic untwisting number equals algebraic unknotting number}

Our proof that $t u_{a}(K)=u_{a}(K)$ generalizes [Borodzik and Friedl 2014; 2015]. Following [Borodzik and Friedl 2014], define a knot invariant $n(K)$ to be the minimum size of a square hermitian matrix $A(t)$ over $\mathbb{Z}\left[t^{ \pm 1}\right]$ such that $\lambda(A)$ is isometric to $\lambda(K)$ and $A(1)$ is congruent over $\mathbb{Z}$ to a diagonal matrix which has only \pm 1 entries. Borodzik and Friedl showed that $u_{a}(K)=n(K)$. Since $t u_{a}(K) \leq u_{a}(K)$, it is obvious that $t u_{a}(K) \leq n(K)$ as well. After stating Borodzik and Friedl's results, we will show that $n(K) \leq t u_{a}(K)$; hence $t u_{a}(K)=n(K)=u_{a}(K)$ for all knots $K$. In fact, we will show something stronger.

Theorem 3.1. Let $K \subset S^{3}$ be a knot. For every algebraic unknotting sequence for $K$ with $u_{+}$positive crossing changes and $u_{-}$negative crossing changes, there exists an algebraic untwisting sequence for $K$ with $u_{+}$positive generalized crossing changes and $u_{-}$negative generalized crossing changes. In particular, $u_{a}(K)=t u_{a}(K)$.

In order to prove Theorem 3.1, we must first recall some notation and results used in [Borodzik and Friedl 2015]. The main theorem of that paper implies that $n(K) \leq u_{a}(K)$ :

Theorem 3.2 [Borodzik and Friedl 2015, Theorem 1.1]. Let $K$ be a knot which can be changed into an Alexander polynomial-one knot by a sequence of $u_{+}$positive crossing changes and $u_{-}$negative crossing changes. Then there exists a hermitian matrix $A(t)$ of size $u_{+}+u_{-}$over $\Lambda$ such that

(1) $\lambda(A(t))$ is isometric to $\lambda(K)$;

(2) A(1) is a diagonal matrix such that $u_{+}$diagonal entries are equal to -1 and $u_{-}$diagonal entries are equal to 1.

In particular, $n(K) \leq u_{a}(K)$.

We need one definition:

Definition 3.3. Let $K$ be a knot and $M(K)$ the result of 0 -surgery on $K$. A 4-manifold $W$ tamely cobounds $M(K)$ if

(1) $\partial W=M(K)$;

(2) the inclusion induced map $H_{1}(M(K) ; \mathbb{Z}) \rightarrow H_{1}(W ; \mathbb{Z})$ is an isomorphism;

(3) $\pi_{1}(W)=\mathbb{Z}$.

If, in addition, the intersection form on $\mathrm{H}_{2}(W ; \mathbb{Z})$ is diagonalizable, we say that $W$ strictly cobounds $M(K)$.

Theorem 3.4 [Borodzik and Friedl 2015, Theorem 2.6]. Let $K$ be a knot and let $W$ be a topological 4-manifold which tamely cobounds $M(K)$. Then $H_{2}(W ; \Lambda)$ is free of rank $b_{2}(W)$. Moreover, if $B$ is an integral matrix representing the ordinary 
intersection pairing of $W$, then there exists a basis $\mathcal{B}$ for $\mathrm{H}_{2}(W ; \Lambda)$ such that the matrix $A(t)$ representing the twisted intersection pairing with respect to $\mathcal{B}$ satisfies

(1) $\lambda(A(t))$ is isometric to $\lambda(K)$;

(2) $A(1)=B$.

We generalize Theorem 3.2 as follows:

Theorem 3.5. Let $K$ be a knot which can be changed into an Alexander polynomialone knot by a sequence of $u_{+}$positive and $u_{-}$negative generalized crossing changes. Then there exists a hermitian matrix of size $u_{+}+u_{-}$over $\Lambda$ with the properties

(1) $\lambda(A(t))$ is isometric to $\lambda(K)$;

(2) A(1) is a diagonal matrix such that $u_{+}$diagonal entries are equal to -1 and $u_{-}$ diagonal entries are equal to 1.

In particular, $n(K) \leq t u_{a}(K)$.

The proof of Theorem 3.5 is similar to that of Theorem 3.2. By Theorem 3.4, in order to prove Theorem 3.5, we only need to show the following proposition.

Proposition 3.6. Let $K$ be a knot such that $u_{+}$positive generalized crossing changes and $u_{-}$negative generalized crossing changes turn $K$ into an Alexander polynomial-one knot. Then there exists an oriented topological 4-manifold $W$ which strictly cobounds $M(K)$. Moreover, the intersection pairing on $\mathrm{H}_{2}(W ; \mathbb{Z})$ is represented by a diagonal matrix of size $u_{+}+u_{-}$such that $u_{+}$entries are equal to -1 and $u_{-}$entries are equal to +1 .

Proof. Let $K$ be a knot such that $u_{+}$positive generalized crossing changes and $u_{-}$ negative generalized crossing changes turn $K$ into an Alexander polynomial-one knot $J$. We write $s=u_{+}+u_{-}$and $n_{i}=-1$ for $i=1, \ldots, u_{+}$and $n_{i}=1$ for $i=u_{+}+1, \ldots, u_{+}+u_{-}$. Then there exist simple closed curves $c_{1}, \ldots, c_{s}$ in $S^{3}-N(J)$ such that

(1) $c_{1} \cup \cdots \cup c_{s}$ is the unlink in $S^{3}$;

(2) the linking numbers $\operatorname{lk}\left(c_{i}, K\right)$ are zero for all $i$;

(3) the image of $J$ under the $n_{i}$-surgeries is the knot $K$.

Note that the curves $c_{1}, \ldots, c_{s}$ lie in $S^{3}-N(J)$; hence we can view them as lying in $M(J)$. The manifold $M(K)$ is then the result of $n_{i}$-surgery on all the $c_{i} \subset M(J)$, where $i=1, \ldots, s$.

Since $J$ is a knot with trivial Alexander polynomial, by Freedman's theorem [Freedman and Quinn 1990] $J$ is topologically slice and there exists a locally flat slice disk $D \subset B^{4}$ for $J$ such that $\pi_{1}\left(B^{4}-D\right)=\mathbb{Z}$. Let $X:=B^{4}-N(D)$. Then $X$ is an oriented topological 4-manifold such that

(1) $\partial X \cong M(J)$ as oriented manifolds; 
(2) $\pi_{1}(X) \cong \mathbb{Z}$

(3) the inclusion induced map $H_{1}(M(J) ; \mathbb{Z}) \rightarrow H_{1}(X ; \mathbb{Z})$ is an isomorphism;

(4) $H_{2}(X ; \mathbb{Z})=0$.

Let $W$ be the 4-manifold which is obtained by adding 2-handles along $c_{1}, \ldots, c_{s} \subset$ $M(J)$ with framings $n_{1}, \ldots, n_{s}$ to $X$. Then $\partial W \cong M(K)$ as oriented manifolds. From now on, we write $M:=M(K)$. Since the curves $c_{1}, \ldots, c_{s}$ are nullhomologous, the map $H_{1}(M ; \mathbb{Z}) \rightarrow H_{1}(W ; \mathbb{Z})$ is an isomorphism and $\pi_{1}(W) \cong \mathbb{Z}$. It thus remains to prove the following lemma:

Lemma 3.7. The ordinary intersection pairing on $W$ is represented by a diagonal matrix of size $s=u_{+}+u_{-}$with $u_{+}$diagonal entries equal to -1 and $u_{-}$diagonal entries equal to 1 .

Recall that the curves $c_{1}, \ldots, c_{s}$ form the unlink in $S^{3}$ and that the linking numbers $\operatorname{lk}\left(c_{i}, J\right)$ are zero. Therefore, the curves $c_{1}, \ldots, c_{s}$ are also nullhomologous in $M(J)$. Thus we can now find disjoint surfaces $F_{1}, \ldots, F_{s}$ in $M(J) \times[0,1]$ such that $\partial F_{i}=c_{i} \times\{1\}$. By adding the cores of the 2-handles attached to the $c_{i}$, we obtain closed surfaces $C_{1}, \ldots, C_{s}$ in $W$. It is clear that $C_{i} \cdot C_{j}=0$ for $i \neq j$ and $C_{i} \cdot C_{i}=n_{i}$.

We argue using Mayer-Vietoris that the surfaces $C_{1}, \ldots, C_{s}$ present a basis for $H_{2}(W ; \mathbb{Z})$. Write $W:=X \cup H$, where $H \cong \bigsqcup_{i=1}^{s}\left(B^{2} \times B^{2}\right)$ is the set of 2-handles attached to $c_{1}, \ldots, c_{s}$. Then write $Y:=X \cap H$, so that

$$
Y=\bigsqcup_{i=1}^{s} N\left(c_{i}\right) \cong \bigsqcup_{i=1}^{s}\left(S^{1} \times D^{2}\right) .
$$

We have the Mayer-Vietoris sequence

$\cdots \rightarrow H_{2}(X) \oplus H_{2}(H) \stackrel{\psi_{*}}{\longrightarrow} H_{2}(W) \stackrel{\partial_{*}}{\longrightarrow} H_{1}(Y) \stackrel{\phi_{*}}{\longrightarrow} H_{1}(X) \oplus H_{1}(H) \stackrel{\psi_{*}}{\rightarrow} H_{1}(W) \rightarrow 0$.

Now, since $H_{1}(Y)$ is generated by all the $S^{1}$-factors, or the longitudes $c_{1}, \ldots, c_{s}$, and $H_{1}(H)=H_{2}(H)=H_{2}(X)=0$, the sequence becomes

$$
0 \rightarrow H_{2}(W) \stackrel{\partial_{*}}{\longrightarrow}\left\langle c_{1}, \ldots, c_{S}\right\rangle \stackrel{i_{*}}{\longrightarrow} H_{1}(X) \stackrel{\psi_{*}}{\longrightarrow} H_{1}(W) \rightarrow 0 .
$$

From [Livingston 1993, Lemma 8.12], for example, we have:

Lemma 3.8. Suppose that for some knot $K$ in $S^{3}$, there is a locally flat surface $F$ in $B^{4}$ with $F \cap S^{3}=\partial F \cap S^{3}=K$. Then the inclusion map induces an isomorphism $H_{1}\left(S^{3}-K\right) \rightarrow H_{1}\left(B^{4}-F\right) \cong \mathbb{Z}$.

In our case, the inclusion $S^{3}-K \hookrightarrow X$ induces an isomorphism $H_{1}\left(S^{3}-K\right) \rightarrow$ $H_{1}(X)$. Since $i_{*}$ is induced by inclusion and the longitudes $c_{1}, \ldots, c_{s}$ are nullhomologous in $S^{3}-K$, we see that $i_{*}$ must be the zero map in $X$. Hence $\partial_{*}$ is an isomorphism $H_{2}(W) \cong H_{1}(Y)$, and $H_{2}(W)=\left\langle C_{1}, \ldots, C_{s}\right\rangle$. 
In particular, the intersection matrix on $W$ with respect to this basis is given by $\left(C_{i} \cdot C_{j}\right)$, i.e., it is a diagonal matrix such that $u_{+}$diagonal entries are equal to -1 and $u_{-}$diagonal entries are equal to +1 . This concludes the proof of Lemma 3.7. Proposition 3.6 follows. Together with Theorem 3.4, this completes the proof of Theorem 3.5.

We have shown that, for every untwisting sequence for $K$ with $u_{+}$positive generalized crossing changes and $u_{-}$negative generalized crossing changes, there exists a hermitian matrix $A(t)$ of size $u_{+}+u_{-}$such that $\lambda(A(t))$ is isometric to $\lambda(K)$ and $A(1)$ is diagonal with $u_{+}$entries equal to -1 and $u_{-}$entries equal to 1. Borodzik and Friedl [2014] have already shown that, for every hermitian matrix $A(t)$ representing $\lambda(K)$ such that $A(1)$ is diagonal with $u_{+}-1$ 's and $u_{-}$ +1 's, there exists an algebraic unknotting sequence for $K$ consisting of $u_{+}$positive and $u_{-}$negative crossing changes. Theorem 3.1 follows.

\section{Untwisting number does not equal unknotting number}

Although the algebraic versions of $t u$ and $u$ are equal, $t u \neq u$ in general. We use a result of Miyazawa [1998] to give our first example of a knot $K$ with $t u(K)=1$ but $u(K)>1$.

Theorem 4.1. Let $K$ be the knot resulting from blowing down the +1 -framed unknot $U \subset S^{3} \backslash V$ in Figure 3. Then $t u(K)=1$ but $u(K)>1$.

From this point forward, we will denote the signature of any knot $K$ by $\sigma(K)$. In order to analyze the unknotting number of $K$, we will use the following theorem:

Theorem 4.2 [Miyazawa 1998]. If $u(K)=1$ and $\sigma(K)= \pm 2$, then

$$
V_{K}^{(1)}(-1) \equiv 24 a_{4}(K)-\frac{1}{8} \sigma(K)(\operatorname{det} K+1)(\operatorname{det} K+5)(\bmod 48),
$$

where $V_{K}^{(1)}$ denotes the first derivative of the Jones polynomial of $K$ and $a_{4}$ is the coefficient of $z^{4}$ in the Conway polynomial $\nabla_{K}(z)=\sum_{n=0}^{\infty} a_{2 n}(K) z^{2 n}$.

We compute using the Mathematica package KnotTheory (http://katlas.org/wiki/ The_Mathematica_Package_KnotTheory) that $\sigma(K)=2$; therefore Theorem 4.2 applies. We also compute using the KnotTheory package that the Jones polynomial $V_{K}(q)$ for our knot $K$ is

$$
V_{K}(q)=q-q^{2}+2 q^{3}-q^{4}+q^{6}-q^{7}+q^{8}-q^{9}-q^{12}+q^{13} ;
$$

hence $V_{K}^{(1)}(-1)=8$. The Conway polynomial of $K$ is computed to be

$$
\nabla_{K}(z)=\sum_{n=0}^{\infty} a_{2 n}(K) z^{2 n}=1+z^{2}
$$


(hence $a_{4}=0$ ), and the determinant of $K$ is 3 . In our case, the right-hand side of the congruence in Theorem 4.2 becomes

$$
0-\frac{1}{4}(4)(8)=-8
$$

and $8 \not \equiv-8(\bmod 48)$. Hence $K$ cannot have unknotting number one, although it was constructed to have untwisting number one. Note that this also shows Miyazawa's Jones polynomial criterion does not extend to untwisting-number-one knots.

\section{Arbitrarily large gaps between unknotting and untwisting numbers}

5A. Arbitrarily large gaps between $u$ and $\boldsymbol{t u}_{\boldsymbol{p}}$. Now that we have shown that there exists a knot $K$ with $t u(K)<u(K)$, it is natural to ask how large the difference $u(K)-t u(K)$ can be. Recall that the $(p, q)$-cable of a knot $K$ is denoted $K_{p, q}$; we denote the $(p, q)$-torus knot by $U_{p, q}$, the $(p, q)$-cable of the unknot. The knots we will be working with are $(p, q)$-cables of knots $K$ with $u(K)=1$ and $\tau(K)>0$, where $p, q>0$.

To get a lower bound on $u\left(K_{p, q}\right)$ for such knots, we compute $\tau\left(K_{p, q}\right)$ for all $p, q$. For cables of alternating (or more generally, "homologically thin") knots such as the trefoil, Petkova [2013] gives a formula for computing $\tau$. However, since we will later compute $\tau$ for cables of nonalternating knots, we use a more general method of computing $\tau\left(K_{p, q}\right)$ using the $\epsilon$-invariant $\epsilon(K) \in\{-1,0,1\}$ introduced by Hom:

Theorem 5.1 [Hom 2014]. Let $K \subset S^{3}$.

(1) If $\epsilon(K)=1$, then $\tau\left(K_{p, q}\right)=p \tau(K)+\frac{1}{2}(p-1)(q-1)$.

(2) If $\epsilon(K)=-1$, then $\tau\left(K_{p, q}\right)=p \tau(K)+\frac{1}{2}(p-1)(q+1)$.

(3) If $\epsilon(K)=0$, then $\tau(K)=0$ and

$$
\tau\left(K_{p, q}\right)=\tau\left(U_{p, q}\right)= \begin{cases}\frac{1}{2}(p-1)(q+1), & q<0, \\ \frac{1}{2}(p-1)(q-1), & q>0 .\end{cases}
$$

Theorem 5.2 [Ozsváth and Szabó 2003]. For the $(p, q)$-torus knot $U_{p, q}$ with $p, q>0, \tau$ equals the 3-sphere genus of $U_{p, q}$, denoted $g\left(U_{p, q}\right)$ :

$$
\tau\left(U_{p, q}\right)=g\left(U_{p, q}\right)=\frac{1}{2}(p-1)(q-1) .
$$

Proposition 5.3 [Hom 2014]. Let $K \subset S^{3}$ be a knot. If $|\tau(K)|=g(K)$, then $\epsilon(K)=\operatorname{sgn} \tau(K)$.

Theorem 5.4. Let $K$ be a knot in $S^{3}$ with unknotting number one. If $\tau(K)>0$ and $p, q>0$, then

$$
u\left(K_{p, q}\right)-t u_{p}\left(K_{p, q}\right) \geq p-1 .
$$

In particular, $t u_{p}\left(K_{p, 1}\right)=1$, while $u\left(K_{p, 1}\right) \geq p$. 
Proof. Let $V$ be the unknot that results from performing the unknotting crossing change on $K$. Consider a generalized crossing change diagram for $V$ together with the \pm 1 -framed surgery curve $U$ that transforms $V$ back into $K$. Then take the $(p, q)$-cable $V_{p, q}$ of $V$ in this diagram, leaving $U$ alone. The resulting $V_{p, q}$ is the $(p, q)$-torus knot before performing the \pm 1 -surgery, but the image of $V$ under \pm 1 -surgery on $U$ is $K$; hence the image of $V_{p, q}$ under the \pm 1 -surgery on $U$ is $K_{p, q}$. Therefore, blowing down the surgery curve $U$ (through which $V_{p, q}$ passes $2 p$ times) results in a diagram for $K_{p, q}$ in $S^{3}$. Since $K_{p, q}$ and $V_{p, q}$ differ by a single twist,

$$
t u_{p}\left(K_{p, q}\right) \leq t u_{p}\left(V_{p, q}\right)+1 .
$$

Since

$$
t u_{p}\left(V_{p, q}\right) \leq u\left(V_{p, q}\right)=\frac{1}{2}(p-1)(q-1),
$$

we get that

$$
t u_{p}\left(K_{p, q}\right) \leq \frac{1}{2}(p-1)(q-1)+1 .
$$

In particular, this inequality shows that $t u_{p}\left(K_{p, 1}\right)=1$. If $\tau(K)>0$, then necessarily $\epsilon(K) \neq 0$ by (3) of Theorem 5.1, so that $\epsilon(K)= \pm 1$. In this case,

$$
\tau\left(K_{p, q}\right)=p \tau(K)+\frac{1}{2}(p-1)(q \mp 1),
$$

and thus

$$
u\left(K_{p, q}\right) \geq\left|\tau\left(K_{p, q}\right)\right|=p \tau(K)+\frac{1}{2}(p-1)(q \mp 1) \geq p+\frac{1}{2}(p-1)(q \mp 1) .
$$

When $q=1$, we get that $u\left(K_{p, 1}\right) \geq p$. Combining our estimates,

$$
\begin{aligned}
u\left(K_{p, q}\right)-t u_{p}\left(K_{p, q}\right) & \geq\left(p+\frac{1}{2}(p-1)(q \mp 1)\right)-\left(1+\frac{1}{2}(p-1)(q-1)\right) \\
& \geq\left(p+\frac{1}{2}(p-1)(q-1)\right)-\left(1+\frac{1}{2}(p-1)(q-1)\right) \\
& \geq p-1 .
\end{aligned}
$$

5B. Arbitrarily large gaps between $\boldsymbol{u}$ and $\boldsymbol{t}_{\boldsymbol{q}}$. The above examples $\left\{K_{p, 1}\right\}$ show that for every $p$ there exists a knot $K_{p, 1}$ with $u\left(K_{p, 1}\right) \geq p$, even though $t u_{p}\left(K_{p, 1}\right)=1$. However, in order to untwist any such $K_{p, 1}$, we must twist at least $2 p$ strands at once. A natural follow-up question is whether there exists a knot $K$ with $u(K) \geq p$ that can be untwisted by a single $\pm q$-generalized crossing change, where $q<p$. More generally, we may ask whether, for any fixed $q$, there is a family of knots which give us arbitrarily large gaps between $u$ and $t u_{q}$. We answer this question in the affirmative.

Theorem 5.5. Let $K$ be a knot with $u(K)=1$ and $\tau(K)>0$, and let $J_{p}^{q}:=\#^{p} K_{q, 1}$. For any $p>0$ and $q>1$, we have $t u_{q}\left(J_{p}^{q}\right) \leq p$ and $u\left(J_{p}^{q}\right)-t u_{q}\left(J_{p}^{q}\right) \geq p$.

Proof. First, we note that for any knot $K, J_{p}^{q}=\#^{p} K_{q, 1}$ can be unknotted by performing $p$ generalized crossing changes on at most $2 q$ strands each, one generalized 
crossing change to unknot each copy of $K_{q, 1}$. Therefore, $t u_{q}\left(J_{p}^{q}\right) \leq p$. Since $\tau$ is additive under connected sum,

$$
\tau\left(J_{p}^{q}\right)=p \cdot \tau\left(K_{q, 1}\right) \geq p q,
$$

and hence $u\left(J_{p}^{q}\right) \geq p q$ for all $p$. Therefore,

$$
u\left(J_{p}^{q}\right)-t u_{q}\left(J_{p}^{q}\right) \geq p q-p=p(q-1) \geq p,
$$

Note. In the case where $K$ has $\sigma(K)= \pm 2$, e.g., when $K$ is a right-handed trefoil knot, we can do better by computing $t u_{q}$ precisely. We use the fact that $\frac{1}{2}|\sigma(K)|$ is a lower bound for $t u_{q}(K)$ for any $q$. First, recall that the Tristram-Levine signature function of a knot $K, \sigma_{\omega}(K)$, is equal to the signature of the matrix $(1-\omega) V+(1-\bar{\omega}) V^{T}$, where $\omega \in \mathbb{C}$ has norm 1 and $V$ is a Seifert matrix for $K$. Note that

$$
\sigma_{-1}(K)=\sigma\left(2\left(V+V^{T}\right)\right)=\sigma\left(V+V^{T}\right)=\sigma(K) .
$$

We use Litherland's formula [1979] for Tristram-Levine signatures of cable knots to compute that

$$
\sigma_{-1}\left(K_{p, q}\right)=\sigma_{(-1)^{p}}(K)+\sigma_{-1}\left(U_{p, q}\right)
$$

and, since $\sigma_{1} \equiv 0$, while $\sigma_{-1}=\sigma$,

$$
\sigma\left(K_{q, 1}\right)= \begin{cases}\sigma(K)+\sigma\left(U_{q, 1}\right)=\sigma(K), & q \text { odd, } \\ \sigma\left(U_{q, 1}\right)=0, & q \text { even, }\end{cases}
$$

since the $(q, 1)$-torus knot is the unknot for any $q$. Now, since the knot signature is additive over connected sum,

$$
\sigma\left(J_{p}^{q}\right)=p \sigma\left(K_{q, 1}\right)= \begin{cases}\sigma(K) \cdot p= \pm 2 p, & q \text { odd } \\ 0, & q \text { even },\end{cases}
$$

and therefore, when $p$ is odd,

$$
t u_{q}\left(J_{p}^{q}\right) \geq \frac{1}{2}\left|\sigma\left(\kappa_{p}^{q}\right)\right|=p .
$$

Since we already know $t u_{q}\left(J_{p}^{q}\right) \leq p$, in fact we must have $t u_{q}\left(J_{p}^{q}\right)=p$ for odd $p \geq 1$.

5C. Arbitrarily large gaps between $u$ and $t u_{q}$ for topologically slice knots. Consider the diagram of an unknot $U(K)$ in Figure 6 , where $K$ is any knot with $\tau(K)>0$. Let $p \geq 2$ be an integer.

We take the $(q, 1)$-cable of $U(K)$, which is still an unknot. Then, we perform a -1-twist on the +1 -framed unknot, obtaining a knot $S^{q}$. Clearly, $t u_{q}\left(S^{q}\right)=1$.

Furthermore, $S^{q}$ is the $(q, 1)$-cable of the knot $D_{+}(K, 0)$, the untwisted Whitehead double of $K$. This is because $U(K)$ represents $D_{+}(K, 0)$ in the manifold obtained from the +1 -surgery, and the cabling operation converts this knot into the 




Figure 6. The knot $U(K)$ (an unknot), together with a +1 -surgery curve.

$(q, 1)$-cable of $D_{+}(K, 0)$. Since untwisted Whitehead doubles are topologically (but not necessarily smoothly) slice [Freedman and Quinn 1990], $D_{+}(K, 0)$ is topologically concordant to the unknot. It is well known that, if $K$ is concordant to $J$, then $K_{m, n}$ is concordant to $J_{m, n}$ for all integers $m, n$. Hence $S_{q, 1}$ is also topologically concordant to the unknot $U_{q, 1}$, and therefore $S_{p}$ is topologically slice for all $p$.

Now, define $S_{p}^{q}:=\#^{p} D_{+}(K, 0)$. Connected sums of topologically slice knots are topologically slice; hence $S_{p}^{q}$ is topologically slice. Moreover, as above, we have that $t u_{q}\left(S_{p}^{q}\right) \leq p \cdot t u_{q}\left(S^{q}\right)=p$.

We will now get a lower bound on $u\left(S_{p}^{q}\right)$ and thus show that $u\left(S_{p}^{q}\right)-t u_{q}\left(S_{p}^{q}\right)$ can be arbitrarily large. The Ozsváth-Szabó $\tau$ invariant gives such a lower bound. Thus, we need to compute $\tau\left(S_{p}^{q}\right)$ for all $p, q$.

We show that $\epsilon\left(D_{+}(K, 0)\right)=1$ and hence, applying Theorem 5.1, that

$$
\tau\left(S^{q}\right)=q \tau\left(D_{+}(K, 0)\right) .
$$

We first compute $\tau\left(D_{+}(K, 0)\right)$.

Theorem 5.6 [Hedden 2007]. Let $D_{+}(K, t)$ denote the positive $t$-twisted Whitehead double of a knot $K$. Then

$$
\tau\left(D_{+}(K, t)\right)= \begin{cases}1, & t<2 \tau(K), \\ 0, & \text { otherwise. }\end{cases}
$$

Since $\tau(K)>0$ in our case, $t=0<2 \leq 2 \tau(K)$, and so $\tau\left(D_{+}(K, 0)\right)=1$. Furthermore, as is the case with any Whitehead double, $g\left(D_{+}(K, 0)\right)=1$, so $\left|\tau\left(D_{+}(K, 0)\right)\right|=1=g\left(D_{+}(K, 0)\right)$ and, by Proposition 5.3,

$$
\epsilon\left(D_{+}(K, 0)\right)=\operatorname{sgn} \tau\left(D_{+}(K, 0)\right)=+1 .
$$

We then apply Theorem 5.1 to $S^{q}$ to get that

$$
\tau\left(S^{q}\right)=q \tau\left(D_{+}(K, 0)\right) .
$$


Since $\tau\left(D_{+}(K, 0)\right)=1$, we have that $\tau\left(S^{q}\right)=q$ and, hence, $\tau\left(S_{p}^{q}\right)=p q$. Thus, $u\left(S_{p}^{q}\right) \geq p q$. Therefore,

$$
u\left(S_{p}\right)-t u_{q}\left(S_{p}\right) \geq p q-p=p(q-1) \geq p,
$$

as desired.

\section{Acknowledgements}

I thank my adviser Tim Cochran for his invaluable mentorship and support. Thanks also to Stefan Friedl, Maciej Borodzik, Peter Horn, and Mark Powell for their mentorship, and to Ina Petkova for suggesting that cables of the trefoil would have unknotting number arbitrarily larger than their untwisting number.

\section{References}

[Adams 1994] C. C. Adams, The knot book: an elementary introduction to the mathematical theory of knots, W. H. Freeman, New York, 1994. MR 1266837 Zbl 0840.57001

[Borodzik and Friedl 2014] M. Borodzik and S. Friedl, "On the algebraic unknotting number", Trans. London Math. Soc. 1:1 (2014), 57-84. MR 3296484 Zbl 1322.57010

[Borodzik and Friedl 2015] M. Borodzik and S. Friedl, "The unknotting number and classical invariants, I”, Algebr. Geom. Topol. 15:1 (2015), 85-135. MR 3325733 Zbl 1318.57009

[Freedman and Quinn 1990] M. H. Freedman and F. Quinn, Topology of 4-manifolds, Princeton Mathematical Series 39, Princeton University Press, 1990. MR 1201584 Zbl 0705.57001

[Hedden 2007] M. Hedden, "Knot Floer homology of Whitehead doubles", Geom. Topol. 11 (2007), 2277-2338. MR 2372849 Zbl 1187.57015

[Hillman 2012] J. Hillman, Algebraic invariants of links, 2nd ed., Series on Knots and Everything 52, World Scientific, Hackensack, NJ, 2012. MR 2931688 Zbl 1253.57001

[Hom 2014] J. Hom, "Bordered Heegaard Floer homology and the tau-invariant of cable knots", J. Topol. 7:2 (2014), 287-326. MR 3217622 Zbl 06366498

[Kronheimer and Mrowka 1993] P. B. Kronheimer and T. S. Mrowka, "Gauge theory for embedded surfaces, I”, Topology 32:4 (1993), 773-826. MR 1241873 Zbl 0799.57007

[Kronheimer and Mrowka 1995] P. B. Kronheimer and T. S. Mrowka, "Gauge theory for embedded surfaces, II”, Topology 34:1 (1995), 37-97. MR 1308489 Zbl 0832.57011

[Levine 1977] J. Levine, "Knot modules, I", Trans. Amer. Math. Soc. 229 (1977), 1-50. MR 0461518 Zbl 0653.57012

[Litherland 1979] R. A. Litherland, "Signatures of iterated torus knots", pp. 71-84 in Topology of low-dimensional manifolds (Chelwood Gate, 1977), edited by R. A. Fenn, Lecture Notes in Math. 722, Springer, Berlin, 1979. MR 547456 Zbl 0412.57002

[Livingston 1993] C. Livingston, Knot theory, Carus Mathematical Monographs 24, Mathematical Association of America, Washington, DC, 1993. MR 1253070 Zbl 0887.57008

[Livingston 2002] C. Livingston, “The slicing number of a knot”, Algebr. Geom. Topol. 2 (2002), 1051-1060. MR 1936979 Zbl 1023.57004

[Mathieu and Domergue 1988] Y. Mathieu and M. Domergue, "Chirurgies de Dehn de pente \pm 1 sur certains nœuds dans les 3-variétés”, Math. Ann. 280:3 (1988), 501-508. MR 936325 Zbl 0618.57004 
[Miyazawa 1998] Y. Miyazawa, “The Jones polynomial of an unknotting number one knot”, Topology Appl. 83:3 (1998), 161-167. MR 1606374 Zbl 0920.57001

[Murakami 1990] H. Murakami, “Algebraic unknotting operation”, Questions Answers Gen. Topology 8:1 (1990), 283-292. MR 1043226 Zbl 0704.57004

[Ozsváth and Szabó 2003] P. Ozsváth and Z. Szabó, "Knot Floer homology and the four-ball genus", Geom. Topol. 7:2 (2003), 615-639. MR 2026543 Zbl 1037.57027

[Petkova 2013] I. Petkova, "Cables of thin knots and bordered Heegaard Floer homology", Quantum Topol. 4:4 (2013), 377-409. MR 3134023 Zbl 1284.57014

[Rolfsen 1976] D. Rolfsen, Knots and links, Mathematics Lecture Series 7, Publish or Perish, Berkeley, CA, 1976. MR 0515288 Zbl 0339.55004

Received August 17, 2015. Revised September 29, 2015.

KENAN INCE

DEPARTMENT OF MATHEMATICS

RICE UNIVERSITY

MS-136

P.O. Box 1892

Houston, TX 77251-1892

UNITED STATES

kenan@rice.edu 


\title{
PACIFIC JOURNAL OF MATHEMATICS
}

Founded in 1951 by E. F. Beckenbach (1906-1982) and F. Wolf (1904-1989)

$$
\text { msp.org/pjm }
$$

\section{EDITORS}

\author{
Don Blasius (Managing Editor) \\ Department of Mathematics \\ University of California \\ Los Angeles, CA 90095-1555 \\ blasius@math.ucla.edu
}

\author{
Paul Balmer \\ Department of Mathematics \\ University of California \\ Los Angeles, CA 90095-1555 \\ balmer@math.ucla.edu \\ Robert Finn \\ Department of Mathematics \\ Stanford University \\ Stanford, CA 94305-2125 \\ finn@math.stanford.edu \\ Sorin Popa \\ Department of Mathematics \\ University of California \\ Los Angeles, CA 90095-1555 \\ popa@math.ucla.edu
}

\author{
Vyjayanthi Chari \\ Department of Mathematics \\ University of California \\ Riverside, CA 92521-0135 \\ chari@math.ucr.edu \\ Kefeng Liu \\ Department of Mathematics \\ University of California \\ Los Angeles, CA 90095-1555 \\ liu@math.ucla.edu \\ Igor Pak \\ Department of Mathematics \\ University of California \\ Los Angeles, CA 90095-1555 \\ pak.pjm@gmail.com \\ Paul Yang \\ Department of Mathematics \\ Princeton University \\ Princeton NJ 08544-1000 \\ yang@math.princeton.edu
}

\section{PRODUCTION}

Silvio Levy, Scientific Editor, production@msp.org

\section{SUPPORTING INSTITUTIONS}

ACADEMIA SINICA, TAIPEI

CALIFORNIA INST. OF TECHNOLOGY

STANFORD UNIVERSITY

UNIV. OF BRITISH COLUMBIA

UNIV. OF CALIFORNIA, BERKELEY

UNIV. OF CALIFORNIA, DAVIS

UNIV. OF CALIFORNIA, LOS ANGELES

UNIV. OF CALIFORNIA, RIVERSIDE

UNIV. OF CALIFORNIA, SAN DIEGO

UNIV. OF CALIF., SANTA BARBARA
KEIO UNIVERSITY

MATH. SCIENCES RESEARCH INSTITUTE

NEW MEXICO STATE UNIV.

OREGON STATE UNIV.
Daryl Cooper

Department of Mathematics

University of California

Santa Barbara, CA 93106-3080 cooper@math.ucsb.edu

Jiang-Hua Lu

Department of Mathematics

The University of Hong Kong

Pokfulam Rd., Hong Kong

jhlu@maths.hku.hk

$$
\text { Jie Qing }
$$

Department of Mathematics

University of California

Santa Cruz, CA 95064

qing@cats.ucsc.edu

\author{
UNIV. OF CALIF., SANTA CRUZ \\ UNIV. OF MONTANA \\ UNIV. OF OREGON \\ UNIV. OF SOUTHERN CALIFORNIA \\ UNIV. OF UTAH \\ UNIV. OF WASHINGTON \\ WASHINGTON STATE UNIVERSITY
}

These supporting institutions contribute to the cost of publication of this Journal, but they are not owners or publishers and have no responsibility for its contents or policies.

See inside back cover or msp.org/pjm for submission instructions.

The subscription price for 2016 is US \$/year for the electronic version, and \$/year for print and electronic.

Subscriptions, requests for back issues and changes of subscriber address should be sent to Pacific Journal of Mathematics, P.O. Box 4163, Berkeley, CA 94704-0163, U.S.A. The Pacific Journal of Mathematics is indexed by Mathematical Reviews, Zentralblatt MATH, PASCAL CNRS Index, Referativnyi Zhurnal, Current Mathematical Publications and Web of Knowledge (Science Citation Index).

The Pacific Journal of Mathematics (ISSN 0030-8730) at the University of California, c/o Department of Mathematics, 798 Evans Hall \#3840, Berkeley, CA 94720-3840, is published twelve times a year. Periodical rate postage paid at Berkeley, CA 94704, and additional mailing offices. POSTMASTER: send address changes to Pacific Journal of Mathematics, P.O. Box 4163, Berkeley, CA 94704-0163.

PJM peer review and production are managed by EditFLOW ${ }^{\circledR}$ from Mathematical Sciences Publishers.

PUBLISHED BY

\section{I. mathematical sciences publishers}

nonprofit scientific publishing

http://msp.org/

(C) 2016 Mathematical Sciences Publishers 


\section{PACIFIC JOURNAL OF MATHEMATICS}

Volume $283 \quad$ No. $1 \quad$ July 2016

A New family of simple $\mathfrak{g l}_{2 n}(\mathbb{C})$-modules

JONATHAN NILSSON

Derived categories of representations of small categories over commutative noetherian rings

BENJAMIN ANTIEAU and GREg STEVENSON

Vector bundles over a real elliptic curve

INDRANIL BISWAS and FLORENT SCHAFFHAUSER

$\mathrm{Q}(\mathrm{N})$-graded Lie superalgebras arising from fermionic-bosonic representations

JIN CHENG

Conjugacy and element-conjugacy of homomorphisms of compact Lie groups

YiNGJUE FANG, GANG HAN and BINYONG SUN

Entire sign-changing solutions with finite energy to the fractional Yamabe equation

DANILO GARRIDO and MoniCa Musso

Calculation of local formal Mellin transforms

ADAM GRAHAM-SQUIRE

The untwisting number of a knot

KENAN INCE

A Plancherel formula for $L^{2}(G / H)$ for almost symmetric subgroups

Bent Ørsted and Birgit SPEH

Multiplicative reduction and the cyclotomic main conjecture for $\mathrm{GL}_{2}$

CHRISTOPHER SKINNER

Commensurators of solvable $S$-arithmetic groups

\section{DANIEL STUDENMUND}

Gerstenhaber brackets on Hochschild cohomology of quantum symmetric algebras and their group extensions

SARAH WITHERSPOON and GUODONG ZHOU 